\title{
Philosophiques
}

\section{Les couleurs, la phénoménologie et la grammaire}

\section{Denis Seron}

Volume 42, numéro 2, automne 2015

URI : https://id.erudit.org/iderudit/1034748ar

DOI : https://doi.org/10.7202/1034748ar

Aller au sommaire du numéro

Éditeur(s)

Société de philosophie du Québec

ISSN

0316-2923 (imprimé)

1492-1391 (numérique)

Découvrir la revue

Citer ce document

Seron, D. (2015). Les couleurs, la phénoménologie et la grammaire.

Philosophiques, 42(2), 385-389. https://doi.org/10.7202/1034748ar d'utilisation que vous pouvez consulter en ligne.

https://apropos.erudit.org/fr/usagers/politique-dutilisation/ 


\title{
Les couleurs, la phénoménologie et la grammaire
}

\author{
DENIS SERON \\ Université de Liège
}

\begin{abstract}
Wittgenstein et la philosophie austro-allemande, de Kevin Mulligan, rassemble huit études dont l'ambition affichée est à la fois historique et philosophique. Leur intérêt n'est pas seulement de révéler, avec lucidité et profondeur, des accointances jusqu'ici quasiment inexplorées avec Brentano et ses héritiers proches ou lointains. La question est aussi de savoir quelles positions sont préférables là où elles divergent. Je voudrais d'abord discuter un détail historique qui me fait problème, sans être tout à fait sûr que le désaccord soit plus qu'une question de formulation. Cette première discussion en amènera une seconde dont l'enjeu, philosophique cette fois, est celuici: sur un point précis où Mulligan accorde sa préférence à Wittgenstein, à savoir sur l'a priori, une certaine conception brentanienne me paraît pour ma part préférable. Comme le point me semble philosophiquement central, j'espère apporter par là un peu plus qu'une remarque de détail.
\end{abstract}

\section{Couleurs composées}

Au chapitre VII, "Couleurs ", Mulligan se livre à une analyse pénétrante des convergences (massives) et divergences (non moins massives, du moins à première vue) entre les théories des couleurs de Wittgenstein et de Meinong. Une part du chapitre porte sur la question de savoir s'il existe ou non des couleurs composées. En d'autres termes, deux couleurs différentes peuventelles apparaître en même temps au même endroit, par exemple le rouge et le bleu dans une surface violette? Mulligan décèle chez Meinong, dans ses Bemerkungen über den Farbenkörper und das Mischungsgesetz de I903, ce qui a tout l'air d'une rétractation sur cette question. Meinong, comme le Tractatus, commence par déclarer que "voir ou s'imaginer du rouge et du bleu exactement au même endroit et exactement au même moment, cela est aussi impossible qu'un carré rond». Mais il semble ensuite faire volte-face: "Je reconnais maintenant (nun) un point de vue d'où moi aussi, en un certain sens, je peux concevoir et même faire mienne l'idée d'un violet fait de rouge et de bleu et donc, si on peut dire, mélangé. » Bref, Meinong semble tour à tour endosser et rejeter la thèse de l'impossibilité des couleurs composées. L'explication la plus simple, celle de Mulligan (I64) si je le comprends bien, est naturellement d'évoquer une rétractation.

Le passage des Bemerkungen atteste indiscutablement que quelque chose a changé dans le point de vue de Meinong. Mais la question de savoir ce qui a changé au juste est peut-être moins simple que ne le suggère Mulligan. Pour ma part, je pense plutôt que Meinong n'y abandonne nullement la thèse de l'impossibilité des couleurs composées, mais qu'il la conserve 
tout en reconnaissant à sa négation un sens acceptable. En réalité, pour lui comme pour Brentano, les deux positions correctement comprises ne se contredisent tout simplement pas et elles peuvent être défendues simultanément.

Un point important, sur lequel je reviendrai plus longuement dans la suite, est que le problème des couleurs composées est un cas particulier du problème de l'analyse psychologique largement étudié par Meinong et d'autres brentaniens, à l'exception notable de Husserl. La question est celleci: à supposer qu'un donné phénoménal $A$ est analysé en ses parties $a, b$ et $c$, ces parties sont-elles déjà données dans $A$ ou bien sont-elles produites par l'analyse? S'il y a problème, c'est parce que les deux options semblent irrecevables. Si $a, b$ et $c$ sont déjà présents dans $A$, alors l'analyse n'est pas informative; si elles sont au contraire produites par l'analyse, alors celle-ci ajoute quelque chose à $A$ et donc l'analysans n'est plus identique à l'analysandum! Comme beaucoup d'autres brentaniens, Meinong a choisi la première option avec un aménagement (Beiträge zur Theorie der psychischen Analyse, I 894). Sommairement: les parties sont déjà présentes dans $A$, l'analyse ne modifie pas réellement $A$, mais elle le modifie néanmoins en un certain sens, pour autant qu'elle focalise l'attention sur ses parties. Ce qui a conduit Meinong (Beiträge zur Theorie der psychischen Analyse, p. $35 \mathrm{I}$ ) et d'autres comme Stumpf (Tonpsychologie, I, I883, p. 32 suiv.) à émettre l'hypothèse paradoxale qu'il existe des contenus sensoriels imperceptibles, c'est-à-dire non remarqués.

Cette manière de voir a d'importantes conséquences sur la question des couleurs composées. Car si toute analyse consiste à dégager des parties déjà présentes dans le tout à analyser, alors l'absence des parties dans le tout à analyser rend l'analyse simplement impossible. Or, dit Meinong, «le violet ne nous met pas en présence du rouge avec le bleu ». On ne voit pas le rouge et le bleu dans le violet, donc le violet est inanalysable, simple. C'est la première conception ci-dessus: "Aucune analyse n'est en mesure de trouver le rouge pur et le bleu pur dans le violet, en ceci que dans le violet on n'est pas en présence du rouge avec le bleu, mais ni du rouge ni du bleu et plutôt d'un tiers terme entre le rouge et le bleu.»

Pourtant, la seconde position de Meinong témoigne d'un retour à l'approche compositionnelle. Comme l'indique à raison Mulligan, ce retour trouve sa justification dans l'idée que les relations entre couleurs peuvent être représentées géométriquement. Cette idée, en effet, prescrit minimalement que les couleurs doivent avoir en commun certains caractères, par exemple leur degré de saturation ou de similitude avec le rouge, qui peuvent être notés au moyen de coordonnées dans un espace tridimensionnel. Or l'argument de Meinong, sa "thèse centrale " (I63) selon Mulligan, est que deux objets différents possédant des caractères communs, c'est-à-dire identiques dans une dimension et différents dans une autre, ne peuvent être simples. Toute couleur - et non seulement toute couleur secondaire - doit 
être composée de caractères représentables par des coordonnées dans un espace tridimensionnel, qui peuvent être révélés par l'analyse.

Ces deux conceptions sont-elles vraiment incompatibles? Il me semble que non, et que leur conjonction définit précisément la position de Brentano sur la question des couleurs composées. Pour ma part, il me semble que la position d'ensemble de Meinong est typiquement brentanienne et que la référence à Brentano, manquante dans l'analyse de Mulligan, peut seule la rendre vraiment intelligible.

Dans une conférence de I 893, le point de départ de Brentano était une expérience de pensée bien connue, celle d'un échiquier composé de cases rouges et de cases bleues ("Vom phänomenalen Grün», dans Untersuchungen zur Sinnespsychologie; cf. "Das Kontinuierliche", dans Philosophische Untersuchungen zu Raum, Zeit und Kontinuum). Divisons chaque case en quatre cases à nouveau rouges et bleues, puis répétons l'opération plusieurs fois. En deçà d'un certain seuil, les cases rouges et les cases bleues sont trop petites pour être discernées, et nous ne voyons plus qu'un grand carré uniformément violet. Quelle conclusion en tirer? D'abord - c'est la première conception de Meinong -, nous ne voyons pas le rouge et le bleu dans le violet, nous ne voyons plus qu'un carré uniformément violet. Mais ensuite, il faut bien que nous voyions, en un certain sens de "voir ", les cases rouges et les cases bleues - mettons comme des limites. Car si nous faisons disparaître toutes les cases rouges et bleues, la surface violette disparaît également: donc si la surface violette apparait, les cases rouges et les cases bleues doivent également apparaître. En conclusion, il y a un certain sens de "voir» où nous ne voyons pas le rouge et le bleu dans le violet, et il y en a un autre où il est nécessaire de les voir — d'en avoir une Einsicht. D'une part, c'est l'analyse du concept de violet qui nous fait apparaître le rouge et le bleu; mais d'autre part, le rouge et le bleu doivent déjà être présents dans le violet à analyser. D'une part le violet est simple, il n'est pas composé de rouge et de bleu comme de parties séparables; d'autre part il est néanmoins analysable - ou "quasi-analysable », pour parler comme Carnap et les gestaltistes berlinois - en rouge et en bleu.

Bien sûr, Meinong ne dirait pas que le violet est composé de rouge et de bleu. Mais son analyse en termes de coordonnées dans l'espace chromatique aboutit à un résultat analogue. Les coordonnées chromatiques ne sont pas ce genre de choses dont il est possible d'avoir des perceptions visuelles comme nous en avons des couleurs elles-mêmes. Les couleurs, affirme Meinong, consistent en "éléments chromatiques en soi non représentables " (für sich unvorstellbare Farbenelemente) (Bemerkungen über den Farbenkörper und das Mischungsgesetz, p. 23). Aussi ces éléments ne sont-ils pas des éléments proprement dits, des parties séparables; ils ressemblent plutôt, comme le souligne très justement Mulligan, à des «moments» (au sens husserlien). Pour l'essentiel, ce sont des "parties distinctionnelles» au sens de Brentano. Le violet, bien que simple, demeure analysable, à savoir distinctionnelle- 
ment ou conceptuellement analysable. (Pour reprendre un exemple de Brentano (Deskriptive Psychologie, p. I3), cette manière de voir met le psychologue descriptif dans une situation analogue à celle de l'atomiste métaphysique quand il parle de moitiés d'atome: la thèse que les atomes ne sont pas composés (de moitiés d'atome) n'empêche assurément pas de parler avec vérité de moitiés d'atome!)

\section{Phénoménologie et analyse conceptuelle}

Si ces éléments me paraissent importants pour la présente discussion, c'est parce qu'ils peuvent conduire à atténuer un peu - sans l'effacer - le contraste entre Wittgenstein et la "phénoménologie».

Mulligan insiste à maintes reprises sur le fait que "Wittgenstein n'a jamais été phénoménologue, pas même lorsqu'il disait vers I930 que son travail relevait de la "phénoménologie" " ( I 52 ). Avec raison, il souligne que s'il est de part et d'autre question de description, le langage et le concept y assument des fonctions différentes, plus ou moins fondamentales: "Alors que ses prédécesseurs conçoivent la description comme portant avant tout sur la complexité des états et des épisodes mentaux et psychologiques ainsi que sur leurs objets, Wittgenstein considère qu'il décrit le langage et la façon dont on emploie les mots» (47). Des constatations analogues peuvent être faites au sujet des essentialismes husserlien ou meinongien. Wittgenstein parle de concepts là où Husserl parle d'essences (39). Pour Wittgenstein, la théorie des couleurs est a priori parce qu'elle est une affaire de logique; pour Meinong, elle l'est parce qu'elle traite de la Beschaffenheit des couleurs.

Dans cette optique, on ne peut que suivre Mulligan lorsqu'il juge inférieure parce que "confuse" l'analyse meinongienne en termes de Beschaffenheit (I56). Mais le point que je souhaite mettre en avant est que le Brentano tardif et peut-être déjà la Psychologie proposent un intéressant medius terminus entre ces deux attitudes. Dans la Deskriptive Psychologie et surtout dans le Versuch über die Erkenntnis, Brentano défend en substance la position suivante. (I) D'abord, la notion kantienne de synthétique a priori est absurde, et tout a priori est en réalité analytique, c'est-à-dire conceptuel. (2) La pensée conceptuelle s'accompagne d'intuitions par lesquelles nous saisissons des relations nécessaires d'inséparabilité ou d'incompatibilité, qui sont en ce sens purement conceptuelles. (3) Cependant, la conception kantienne nous induit encore en erreur en nous faisant croire qu' «a priori» s'opposerait à «empirique ». Il n'en est rien, pour deux raisons. La première est que, pour Brentano, tous les concepts sont empiriques, c'est-à-dire soit directement abstraits de l'expérience, soit obtenus par combinaison de concepts directement abstraits de l'expérience. La seconde raison est qu'un concept est un phénomène mental, c'est-à-dire quelque chose dont nous avons des expériences internes et qui, en tant que tel, se prête comme les autres phénomènes mentaux à l'analyse psychologique. 
Par exemple, on peut supposer qu'il est nécessaire qu'un homme ait ni plus ni moins qu'un père biologique. Mais de quelle nature est cette nécessité ? Est-elle, en un sens mystérieux, une nécessité biologique? Ou bien une nécessité conceptuelle ou grammaticale, qui tient à l'usage du mot "père »? La conception brentanienne permet d'affirmer les deux sans incohérence: cette nécessité est à la fois biologique et conceptuelle. Elle est intrinsèque au concept et en ce sens analytique, mais elle est empirique au sens où le concept est un concept empirique: il aurait certes été différent si l'expérience avait été différente, mais il n'en renferme pas moins des relations a priori. Ainsi, le psychologue ne cesse pas d'être empiriste quand il énonce des lois a priori, quand il affirme que sont impossibles un violet sans nuance de rouge, un jugement sans présentation ou sans affirmation ni négation.

Il y a des raisons de penser que cette manière de voir était déjà quoique sous une forme peu développée - celle de la Psychologie de I874. "Notre but, écrivait alors Brentano, est de rendre distincts les deux expressions "phénomène physique" et "phénomène psychique". Nous voulons dissiper toutes les mécompréhensions et confusions à leur sujet » (Psychologie vom empirischen Standpunkt, I973, p. I I I). Par ailleurs, la possibilité d'analyser en parties inséparables - distinctionnelles, abstraites, irréelles - est un réquisit essentiel de la psychologie brentanienne déjà en I 874 . Après tout, la thèse brentanienne de l'intentionnalité équivaut à affirmer que nécessairement les plus petites parties (séparables) obtenues par analyse d'un acte mental sont toujours des combinaisons d'une intentio et d'un intentum, d'un objet primaire et d'un objet secondaire, lesquels sont donc des parties inséparables obtenues par abstraction (Psychologie vom empirischen Standpunkt, I973, p. I79).

Quoi qu'il en soit, cette conception me semble cette fois donner l'avantage à l'approche "phénoménologique ». Loin de l'obscur essentialisme husserlien et meinongien, mais aussi d'un certain mystérianisme linguistique wittgensteinien, Brentano apporte bien une réponse à la question "d'où vient la grammaire?». Et sa réponse est celle-ci: de l'expérience, encore et toujours de l'expérience. 